\title{
The role of disciplinary perspectives in an epistemology of scientific models
}

\author{
Mieke Boon ${ }^{1}$ (D)
}

Received: 21 October 2019 / Accepted: 4 June 2020 / Published online: 1 July 2020

(C) The Author(s) 2020

\begin{abstract}
The purpose of this article is to develop an epistemology of scientific models in scientific research practices, and to show that disciplinary perspectives have crucial role in such an epistemology. A transcendental (Kantian) approach is taken, aimed at explanations of the kinds of questions relevant to the intended epistemology, such as "How is it possible that models provide knowledge about aspects of reality?" The approach is also pragmatic in the sense that the questions and explanations must be adequate and relevant to concrete scientific practice. First it is explained why the idea of models as representations in terms of similarity or isomorphism between a model and its target is too limited as a basis for this epistemology. An important finding is that the target-phenomenon is usually not something that can be observed in a straightforward manner, but requires both characterization in terms of measurable variables and subsumption under (scientific) concepts. The loss of this basis leads to a number of issues, such as: how can models be interpreted as representations if models also include conceptually meaningful linguistic content; how can researchers identify non-observable real-world target-phenomena that are then represented in the model; how do models enable inferential reasoning in performing epistemic tasks by researchers; and, how to justify scientific models. My proposal is to deal with these issues by analyzing how models are constructed, rather than by looking at ready-made models. Based on this analysis, I claim that the identification of phenomena and the construction of scientific models is guided and also confined by the disciplinary perspective within which researchers in a scientific discipline have learned to work. I propose a Kuhnian framework by which the disciplinary perspective can be systematically articulated. Finally, I argue that harmful forms of subjectivism, due to the loss of the belief that models objectively represent aspects of reality, can be overcome by making the disciplinary perspective(s) in a research project explicit, thereby enabling its critical assessment, for which the proposed Kuhnian framework provides a tool.
\end{abstract}

This article belongs to the Topical Collection: Perspectivism in science: metaphysical and epistemological reflections

Guest Editor: Michela Massimi

Mieke Boon

m.boon@utwente.nl

Extended author information available on the last page of the article 
Keywords Scientific practice $\cdot$ Scientific models $\cdot$ Phenomena $\cdot$ Measurements · Representation · Similarity · Semantic view of theories · Transcendental method · Disciplinary perspectives

\section{Introduction}

This article aims at developing an epistemology of scientific models. The focus is on empirical and experimental research practices that work in the context of concrete societal or (socio-)technological challenges. ${ }^{1}$ I will argue that the role of disciplinary perspectives is crucial to an adequate epistemology of scientific models in these practices.

Traditional philosophy of science focused of the role of models in testing or justifying abstract theories, which has been systematically worked out in the semantic view of theories. I will explicate this as an epistemology of models, and use this as a point of reference for developing an epistemology of scientific models that does more justice to their roles in scientific research practices. The adjective 'scientific' is to emphasize that the intended epistemology is about models that play an independent epistemic role, rather than just serving in the justification of theories. I will argue that an epistemology of scientific models in research practices involves a number of interrelated questions that require philosophical clarification. It begins with the basic question: "What is a scientific model?" In the philosophy of science, a common answer is that a model is a representation of a real-world target-system or phenomenon. ${ }^{2,3}$ This leads to the next question: "What is meant by the idea that models represent a targetphenomenon?" When oriented at scientific practice and the epistemic uses of models, an epistemology of scientific models must also address: "How is it possible that humans gain knowledge about aspects of reality by scientific models?" This points at a more specific question: "How is it possible that scientific models allow for epistemic tasks and inferential reasoning by humans?" Furthermore, assuming that scientific models are used for performing epistemic tasks raises the question: "How are scientific models justified?" It will appear that also the notion of 'the targetphenomena represented by the model' requires attention, in particular when scientific models represent target-phenomena that are not observable in a straight-forward manner. This introduces two additional questions: "What is a phenomenon?" and "How is it possible that models represent non-observable target-phenomena?" Having addressed these questions in the first part of this article, in the second part I will argue that disciplinary perspectives form an inherent part of the proposed epistemology of scientific models. Here, I will propose a Kuhnian framework that enables to systematically articulate and critically evaluate the disciplinary perspective(s) of researchers working in research projects.

In developing an epistemology of scientific models, I adopt a transcendental (Kantian) and pragmatic approach. In this approach the format of asking philosophical

\footnotetext{
${ }^{1}$ My focus on scientific practice includes a normative stance in the sense that philosophical accounts must be adequate and relevant for (specific types of) scientific practice.

2 See Frigg and Hartmann (2018) and Frigg and Nguyen (2016b) for comprehensive overviews.

${ }^{3}$ Alternatively, philosophers have proposed that models are fictions (e.g., Suárez, ed., Suárez 2009a, b). In the present article, I will focus on the idea that models are representations.
} 
questions is: "How is it possible that (for example, models provide knowledge about aspects of reality)?" In other words, "What must be presupposed about scientific practices, the character of epistemic entities (e.g., models), and human cognition to explain that this is possible?" A transcendental approach thus seeks explanations for the "How is it possible ...?" questions such as those raised above. Conversely, the "What is ...?" questions are mostly secondary in the sense of being based on these explanations. ${ }^{4}$ The pragmatic part of my approach is that the questions and explanations must be adequate and relevant to concrete scientific practice.

An overview of the structure and conclusions of this article can be found in Section 7.

\section{The semantic view: models as representations of theories}

\subsection{What are models in the semantic view of theories?}

This section aims to show that the semantic view offers a straightforward account of the representational relationship between scientific models and real-world target-phenomena, but next, that this account is too limited as an epistemology of scientific models in scientific research practices.

In the semantic view, the role assigned to models is subordinate to the question of how abstract theories can be tested. Testing a theory involves combining a 'top-down' and a 'bottom-up' approach (see, Figure 1 in Giere 2010). Top-down, models of imaginary phenomena or systems (for example the ideal harmonic oscillator) are (mathematically) derived from the abstract theory (e.g., axiomatic systems such as Newton's laws of motion). Giere calls them representational models. These representational models are sometimes called representations of the theory but are also referred to as instantiations of the theory (also see Giere 1999, 167-8). Concurrently, these models represent the imaginary phenomenon.

Here I introduce the notion of imaginary phenomena to make a clear distinction with the real-world phenomenon, for example, real-world oscillations. It is worth stressing that the philosophical focus of the semantic view is not primarily on the modeling of a 'real-world' phenomena. Nevertheless, the purpose of the semantic view to explain how theories are tested does require to connect between the imagined phenomenon and a real-world target-phenomenon.

The model derived from the abstract theory usually consists of a set of mathematical equations, which can be plotted in graphs by making calculations that predict model-

\footnotetext{
${ }^{4}$ A Kantian (transcendental) approach can be compared with approaches in science that aim at explanations. In the natural sciences these explanations cannot be 'read' from nature. Therefore, researchers ask what must be presupposed about the (purported) underlying (non-observable) structure of nature to explain observable events. In a similar way, a transcendental approach in philosophy starts from asking what must be presupposed about human cognition and their epistemic strategies to explain epistemic results. Researchers can only assess an explanations for its value to the intended (epistemic or pragmatic) uses. Hence, the suggested similarity between approaches in the natural sciences to look for explanations, on the one hand, and transcendental approaches in philosophy, on the other, agrees to an anti-realist epistemology according to which humans are 'in principle' unable to determine whether the proposed explanation is literally true. This anti-realist (and antimetaphysical) assumption about both science and philosophy guides my approach throughout the present study.
} 
outcomes. Bottom-up, data are generated through real-world experiments, for example, experiments that generate real oscillations and produce data by measuring location or angle as a function of time. On the basis of these experimentally generated data-sets of the real-world phenomenon, so-called data-models are generated by also using theoretical knowledge about the experimental technologies and statistical procedures on 'raw' data. Data-models represent the real-world target-phenomenon. These datamodels can also be plotted in graphs. Subsequently, the test of the theory consists of comparing (e.g., visually) the plotted (non-linguistic) structures that are based 'topdown' on the representational model that represents the imaginary phenomenon under conditions occurring in the experiments, and 'bottom-up' on the plotted data-model that represents the real-world phenomenon at the experimental conditions. ${ }^{5}$ Based on this methodology of comparing theoretically predicted and experimentally generated (nonlinguistic) structures, scientists decide whether the theory meets epistemic criteria such as empirical adequacy (Van Fraassen 1980, 2008).

It is important to note that the semantic view provides an epistemology of abstract theories that draws on the possibility to objectively compare structures. Apparently, one structure can be called the representation of another structure because the semantic relationship between them is (partially) isomorphic. This approach to testing theories only works when using non-linguistic entities. ${ }^{6}$ My aim is not to claim that the semantic view is philosophically unproblematic, but to emphasize that its plausibility is based on the premise of comparable relationships between structures, which is evaluated in terms of semantic notions such as '(partial)-isomorphism,' 'similarity,' 'resemblance,' 'likeness,' or 'mapping.'

\subsection{How are scientific models justified?}

An epistemology of scientific models ${ }^{7}$ requires an explanation of how these models are justified or evaluated, which means that it must be assessed whether they are correct about the real-world target-phenomenon. First, what can we learn from the semantic view about testing scientific models?

The semantic view makes it clear that testing occurs by comparing theoretically generated models (i.e., non-linguistic structures representing the theory) with empirically grounded structures (i.e., non-linguistic structures representing the real-world target-phenomenon). Hence, on the one hand, it is the model-outcomes generated by the non-linguistic structure that is derived from the theory to represent the imaginary

\footnotetext{
${ }^{5}$ Also see Suppe (1989) for a comprehensive explanation of the Semantic View of Theories.

${ }^{6}$ More specifically, a number of representational relationships play a role in this account of testing abstract theories: (a) the representational model representing the abstract theory; (b) the representational model representing the imaginary phenomenon; (c) the real-world phenomenon generated in the experimental setup representing the imaginary phenomenon, vice versa; (d) the raw data representing the real-world phenomena; and (e) the data-model representing the raw data. Each of the sentences (a)-(e) has the form "A represents B." Eventually, the test of the theory is by comparison between two structures: (f) the representational model is partially isomorphic or structurally similar to the data-model, having the form "A is / is not partially isomorphic (or structurally similar) to B." When assuming that the representational relationships (a-e) are (partial) isomorphic relationships between structures, and also, that isomorphic relationships are transitive (i.e., if structure $\mathrm{A}$ is isomorphic to structure $\mathrm{B}$, and $\mathrm{B}$ is isomorphic to $\mathrm{C}$, then $\mathrm{A}$ is also isomorphic to $\mathrm{C}$ ), then this way of reasoning to test or justify the abstract theory is sound.

${ }^{7}$ Instead of representational model (as in the semantic view), I will use the notion scientific model.
} 
phenomenon (e.g., the imaginary harmonic oscillator) at the physical conditions in the experimental set-up, and on the other hand, the data-model generated by an experimental set-up that somehow mimics the imaginary phenomenon. Comparison requires that researchers manage to physically generate the imaginary phenomenon by means of the experimental set-up. Crucially, this involves that the variables that characterize the imaginary phenomenon in the model are the same as the measurable variables in the experimental set-up.

The asset of the semantic view is that comparison between the scientific model and the real-world target-phenomenon merely occurs between (non-linguistic) structures that only make use of the measurable variables (e.g., time, location, angle, length, mass). Hence, the scientific model represents the imagined phenomenon not 'literally' as a picture or photograph, but in terms of a set of measurable variables, while the realworld phenomenon is represented in terms of the same set of measurable variables. Moreover, in the case of non-observable phenomena, the comparison is 'only' made between model-outcomes and data-models - i.e., between a structure generated by the scientific model at conditions in the experimental set-up, on the one hand, and datamodels derived from data measured in an experimental set-up, on the other hand. There is no 'direct' comparison between the scientific model and the purported non-observable real-world phenomenon. ${ }^{8}$

\section{Models as representations of real-world phenomena}

\subsection{Models as mediators and autonomous agents}

Crucial to the semantic view of theories is the idea that scientific models are (mathematically) derived from abstract scientific theories. In that capacity, scientific models are used to justify or test the abstract theory. In the renowned collection Models as Mediators, Morrison and Morgan (eds. 1999) defend a more extended view of models in science. ${ }^{9}$ Their goal is "to clarify at least some of the ways in which models can act as autonomous mediators in the sciences and to uncover the means by which they function as a source of knowledge" (ibid, 8). They introduce the notion of models as mediating instruments, and argue that "if models are to play an autonomous role allowing them to mediate between our theories and the world, and allowing us to learn about one or the other, they require such partial independence" (ibid, 17). Clearly, their ideas are still close to the semantic view when they assume that "models represent either aspects of our theories, or aspects of our world, or more typically aspects of both at once, ... [T] he model represents, in its details, both the theory and a real world pendulum" (ibid, 32). At the same time, they depart from the semantic view when claiming that the model "functions as an autonomous instrument which allows us to

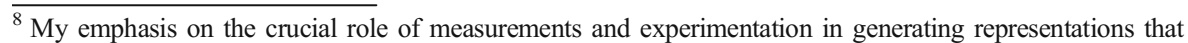
allow for comparison agrees with Van Fraassen's (2008): "it is not only to our understanding of theories and their models that representation is relevant. The achievement of theoretical representation is mediated by measurement and experimentation, in the course of which many forms of representation are involved as well," (ibid, 2, my emphasis).

${ }^{9}$ See Frigg and Hartmann (2018) for a comprehensive overview of philosophical discussions on models in science.
} 
make the correct calculations for measurements to find out a particular piece of information about the world" (ibid, 32).

Likewise, Morrison (1999) assumes that models rather than abstract theory represent and explain the behavior of physical systems. She assumes that models are derived from theories, which accords with the semantic view, but she expands on it by defending that models do so in a way that makes them autonomous agents in the production of scientific knowledge. She explains this idea by an example showing that the model of the boundary layer in fluid mechanics cannot be mathematically derived from the Navier-Stokes equations alone, but also involves phenomenological descriptions and conceptual understanding of viscous flows. Thus, Morrison's analysis shows the crucial role of descriptions and conceptual content in constructing scientific models that cannot be derived from abstract theory only. Therefore, an epistemology of scientific models must take into account the role of linguistic and conceptual content. Yet, this implies that we lose the aforementioned benefits of the semantic view that are based on the assumption that models are non-linguistic entities.

In summary, the idea of models as mediators shows that the semantic view gives a very limited view on the role of models in scientific practice. First, not all models are derived from abstract theories. Secondly, testing abstract theory is not the only epistemic function of models, but also have independent (autonomous) epistemic roles in science. This means that models themselves are sources of knowledge in the sense that models can be used to generate knowledge about the real-world target-phenomenon. Thirdly, models also entail linguistic (conceptually meaningful epistemic) content.

\subsection{How do scientific models represent non-observable target-phenomena?}

The epistemology of scientific models according to the presented interpretation of the semantic view involves that, in the case of non-observable phenomena, the representational relationship exists only between model-outcomes and data-models. More specifically, there is only a representational relationship between the non-linguistic outcomes of the scientific model calculated at conditions in the experimental set-up, and data-models derived from data measured in an experimental set-up that supposedly generates or investigates the purported non-observable phenomenon. On this account, there is not a 'direct' representational relationship in terms of isomorphism or similarity between the scientific model and the purported non-observable phenomenon.

However, current accounts of scientific models do not seem to adopt this very restricted sense in which the notions 'representation,' and 'similarity,' etc. are to be understood. To the contrary, authors often suggest that models are a more or less literal (although idealized), picture-like representations of (non-observable) real-world phenomena. This is illustrated, for instance, in these quotes by Giere (2002): "Models are objects that can be used to represent reality by exhibiting a designated similarity to physical objects ... My prototype for a model is a standard road map. This is a physical object (usually made of paper) that I would say represents a terrain in virtue of quite specific spatial similarities. I move on to scale models, such as Watson's original physical model of DNA." These kinds of metaphors are intelligible when the model represents observable phenomena (e.g., as in graphic art, or in design), or when the representation can be understood as resulting from a specific type of mapping (e.g., 3D to 2D). In these cases, it is possible for knowledgeable researchers to compare the 
model with the target-phenomenon. But such a comparison is hard to imagine when it comes to non-observable phenomena (e.g., as in the model of DNA). I therefore tend to agree with Van Fraassen (2008), who argues that if the meaning of representation involves the idea of likeness or similarity, this can only concern observable phenomena (e.g., ibid., 87). Yet, I disagree with Van Fraassen that phenomena are observable by definition. It is common practice in scientific research to refer to all kinds of nonobservable phenomena.

An epistemology of scientific models should therefor include a comprehensible explanation of the representational relationship between scientific models and nonobservable real-world target-phenomena. This forces us to specify what we mean by phenomena. In particular, how do we identify and specify the target-phenomenon represented in the model? Is the target-phenomenon represented in the model epistemically independent of the model? How does the target-phenomena become known to us? In the case on non-observable phenomena, does this not already involve a scientific model of the phenomenon? All this implies that an epistemology of scientific models also requires an epistemology of phenomena.

\section{An epistemology of observable and unobservable target-phenomena}

\subsection{What is a phenomenon?}

Although there is an extensive literature on phenomena, the question "what is a phenomenon?" is not often discussed in the literature on models. ${ }^{10}$ Therefore, the way in which the concept of phenomenon is used needs clarification. Let us first look at some examples. Well-known historical examples of observable phenomena discovered by scientists are: the orbit of the Moon, the patterns formed in iron filings on paper covering a magnet, the piezo-electric effect, and the emission and absorption spectra in heated hydrogen gas. Commonly, the observed phenomena are represented by drawings or tables or graphs. ${ }^{11}$ In the semantic view, these types of representations are called data or data-models. However, this suggests that the phenomena just described can be reduced to a pattern in data. It suggests that 'observed phenomena' are the observed patterns (or structures) that occur in nature or are generated in experimental setups. But in this manner, we seem to lose essential information. Surely, this account of observable phenomena is adequate to the project of the semantic view. But it does not fully cover the roles of phenomena and their modelling in concrete scientific practices. So, can we come up with an account that is more adequate about these roles?

In this section, I aim for an epistemology of phenomena that is adequate with regard to experimental research and scientific modelling practices. First I will analyze various

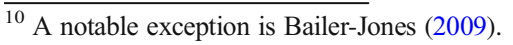

${ }^{11}$ I agree with Van Fraassen (2008) on the view that phenomena created by instruments are observable phenomena: "our instruments are engines of creation. They create new observable phenomena, ones that may never have happened in nature... Those new phenomena are themselves observable, and become part of our world," (ibid, 87).
} 
ideas within the philosophy of science about phenomena. ${ }^{12}$ Next, I will outline an account that I find plausible for solving the various conceptual and epistemological issues that emerge in this analysis. Finally, I will explain in Section 5 how this account of phenomena fits with the ideas proposed in this article about how models are constructed within disciplinary perspectives.

\subsection{What are phenomena in the semantic view?}

I take Van Fraassen as a representative of the interpretation of the semantic view that I endorse. In accordance with his empiricist stance, he assumes that the task of science is to represent the observable phenomena, which he refers to as 'to save the phenomena' (Van Fraassen 2008, 86). This take on the matter implies that not the scientific model, but the model-outcome represents the observable phenomenon. As said, the model-outcome is expected to be (partially) isomorphic to the observed phenomenon at conditions in the experimental setup. It is in this very manner that the model, when correct, represents the observed phenomenon. Clearly, this agrees with how the semantic view explains representational relationships in testing theories. As outline above, observed phenomena in turn, are mere patterns or structures in measured data, which are represented by drawings or tables or graphs, and called data or data-models.

But what does this mean for the content of the scientific model? According to Van Fraassen, "A model often contains much that does not correspond to any observable feature in the domain. Then, from an empiricist point of view, the model's structure must be taken to reveal structure in the observable phenomena, while the rest of the model must be serving that purpose indirectly" (ibid, 87, my emphasis). In my view, Van Fraassen's (anti-realist) empiricist position correctly avoids the suggestion that models can provide literal, picture-like representations of unobservable phenomena. Yet, his view also seems quite empty as to the content of the model. In short, in this view on phenomena, the expression "models represent their target-phenomenon" only means that model-outcomes represent observable phenomena, while the content of the model does not represent anything real. Altogether, this view is insufficient regarding the role of non-observable real-world phenomena in scientific practices.

\subsection{Van Fraassen on observable phenomena}

Van Fraassen's reason to distinguish between observable and non-observable phenomena is an empiricism that "involves a common sense realism in which reference to observable phenomena is unproblematic" (2008, 3). Although I am sympathetic to Van Fraassen's empirical stance, his assumption that "the aim of science is to provide empirically adequate theories about what the world is like [i.e., about observable phenomena]'(ibid, 87), is too limited as an account of scientific practices. I agree that observable phenomena play a crucial role in testing empirical adequacy as a way to justify a theory or scientific model. Yet, the aim of science is not only empirically adequate theories, but also the construction of scientific models that allow different kinds of inferential reasoning. ${ }^{13}$

\footnotetext{
$\overline{12}$ See Chapter 7 in Bailer-Jones (2009) on phenomena, data and data-models.

13 In this article, the notions 'inferential reasoning' (Suárez 2004) and 'epistemic activities' (Chang 2014) are used. Inferential reasoning according to Suárez assumes reasoning upon (non-linguistic) structures, whereas epistemic activities in the sense of Chang is a more open notion. I will use the two notions interchangeably, assuming that inferential reasoning is not limited to reasoning based on structures, but also based on, for example, conceptual content.
} 
In summary, unlike Van Fraassen's point of view, I defend that philosophical accounts of models should not be limited to observable phenomena, but also explain the role of postulated non-observable target-phenomena. In my view, non-observable phenomena are postulated to enable different kinds of inferential reasoning, which do not necessarily have to lead to true results, but which are productive to advance scientific research. $^{14}$

\subsection{The problem of unobservable phenomena}

The other extreme is to assume that a scientific model literally represents an unobservable target-phenomenon. I call this a picture-metaphor of models. A 'literal representation' in this context means something akin to how non-linguistic entities such as photographs, drawings, numerical tables, and graphs represent observable phenomena. As outlined above, authors like Giere suggest that these non-linguistic entities are 'direct' representations in the sense that humans are capable to recognize them as representations in a straightforward manner. Therefore, the expression "models represent their target-phenomenon" in this interpretation means that scientific models represent non-observable phenomena in a more or less literal fashion. Suggestive examples are the model of DNA referred to by Giere, but also the Bohr model of the atom, and models representing proteins and biochemical mechanisms. I stress that, in view of an epistemology of models, this interpretation is highly problematic because it is unclear how to arrive at more or less literal representations of these kinds of unobservable phenomena.

\subsection{Bogen and Woodward's distinction between data and phenomena}

Bogen and Woodward's (1988) defense of the notion of phenomena can be taken as a realist, practice oriented response to Van Fraassen's (1980) anti-realist notion of "saving the phenomena." They defend that phenomena are: distinct from data; objective, stable features of the world; not always observable; not low-level theories; and, inferred from data (also see Bogen 2011; Woodward 2011). Loosely speaking, according to them, data are the observations reported by experimental scientists, while phenomena are objective, stable features of the world to which scientists infer based on reliable data. Data are 'directly observed' and idiosyncratic to particular experimental contexts, whereas phenomena have stable, repeatable characteristics that are detectable by means of a variety of different procedures, which may yield quite different kinds of data. Ontologically and epistemologically, they think of phenomena as being in the world, not just the way we talk about or conceptualize the natural order. On their realist account, therefore, phenomena are physical entities that exist independent of us.

Although Bogen \& Woodward's account of phenomena is intuitively plausible from a scientific practice point of view, the philosophical difficulty is that their empiricism requires reconciling two assumptions, namely, on the one hand, that phenomena are

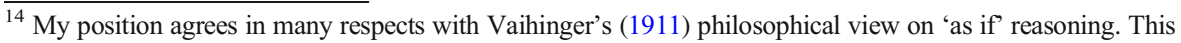
views, in turn, is close to current epistemological interpretations of Kant's philosophy of science that I endorse. Vaihinger's ideas have been taken up in the current movement of models as fictions (e.g., Fine 1993; Suárez $2009 a, b)$. Although relevant to the issues at stake, I will not elaborate on the idea of models and representations as fictions in the present article.
} 
inferred from data, and, on the other hand, that phenomena are not some kind of lowlevel theories. However, inferring phenomena from data, for instance by means of statistical methods, implies that the phenomenon is identical with the data-model derived from the data (Glymour 2002), which does not add much to data-models in the semantic view. The other option is that the phenomenon (or should we rather say, the description of the phenomenon) represents a theoretical or conceptual interpretation of the data, which means that conceptual content has been added so that the data (or datamodel) is converted into a low-level theory, which they explicitly aim to avoid. Below, I propose that Massimi's Kantian take on this issue provides a viable alternative.

\subsection{Phenomena as entities that ask for scientific explanations}

According to Hacking (1983), "[Phenomenon] has a fairly definite sense in the writing of scientists. A phenomenon is noteworthy. A phenomenon is discernible. A phenomenon is commonly an event or process of a certain type that occurs regularly under definite circumstances" (ibid, 221). Hacking refers to observable phenomena as not only occurrences observed in nature but also new phenomena that are generated in experimental set-ups, often through the operation of new technological instruments and noticed by attentive researchers who intervene with this equipment. I agree with Hacking (1983) that science postulates successful non-observable phenomena that are held causally responsible for specific observable phenomena and which he calls 'theoretical entities.' However, I am hesitant about his 'entity realism,' which is why I stick to the expression 'purported non-observable real-world phenomena.' ${ }^{15}$

Accordingly, another option to explain what we mean by 'phenomena' is that scientific models structure or explain rather than 'literally' represent the observed or unobservable phenomenon. Hence, the scientific model can represent the phenomenon in terms of a (logical, morphological, or mathematical) structure that tells more than the data or data-pattern observed in nature or in the laboratory. In addition, a scientific model can also represent an explanation of the observed phenomenon, which usually includes representations of the causal workings in terms of (purported) non-observable real-world phenomena (such as mechanisms). The expression "models represent their target-phenomenon" then means that scientific models represent (purported) 'underlying' structures, that are presupposed to explain the observable phenomena - rather than representations being mere 'descriptions.' Altogether, these different options show that it is not fully clear what we actually mean by the expression that "models represent their target-phenomenon."

\subsection{Massimi: phenomena are not ready-made}

One of the reasons to argue for the role of disciplinary perspectives in science is that phenomena, be it observable or non-observable, are never observed in a straightforward

\footnotetext{
${ }^{15}$ More specifically on the meaning of purported non-observable real-world phenomena: I do not endorse a form or referential realism (see Teller 2020 for a plausible argument against referential realism). Instead, my position agrees in many respects with Van Fraassen's (epistemological) anti-realism. This position emphasizes a common-sense realism, not in the sense of believing that the purported phenomenon has a referent in the real world, but rather in the sense that observations and experiences of researchers derive from their (physical and technological) interactions with a world that is physically independent of their thoughts and beliefs.
} 
manner, but always involve what can be called a perspective (e.g., a mathematical or conceptual framework). To make this point, I turn to Massimi's (2007, 2008, 2011) Kantian account of phenomena. Massimi (2008) engages in the controversy between scientific realists (including Hacking and Giere) and Van Fraassen's anti-realist constructive empiricism by asking, "how do we know that the entities, their properties and relations as described by our best scientific theories truly correspond to the way things are in nature?" (ibid, 1). Massimi argues that a prevailing conception of phenomena, according to which phenomena are what 'appears' to us and to our perceptual apparatus, is at the heart of this debate. Therefore, both scientific realist and constructive empiricist positions are entrapped in "the view that phenomena are empirical manifestations of what there is" (ibid, 3). This view entails the idea that phenomena are 'readymade,' that is, phenomena lay bare in front of us. Massimi believes this view is inadequate. Her purpose is to show that phenomena are not ready-made for a scientific theory to either save them, as Van Fraassen thinks, or give a literally true story of them, as believed by scientific realists.

The alternative philosophical conception of phenomena she proposes goes back to Kant. Massimi argues that from a Kantian point of view phenomena are not ready-made, not mere empirical manifestations of what there is. Instead, phenomena conform to our ways of representing: "our representation of things as they are given to us does not conform to these things as they are in themselves but rather these objects as appearances conform to our way of representing" (ibid, 9, my emphases). Therefore, "[A] phenomenon, ..., is a conceptually determined appearance. ... phenomena are appearances brought under the concepts of the faculty of understanding so as to make experience finally possible," (ibid, 10, 11). Massimi makes this idea about phenomena more concrete as follows: "in nature we may observe objects moving in space and time, changing physical state (from solid to liquid to gaseous) or displaying some properties (e.g. being elastic). But these are only appearances [Erscheinungen]. Only when we introduce moving forces as the underlying causes that make the objects move in space, or change their physical state, or displaying some physical or chemical properties, do we have a conceptually determined appearance or phenomenon as the proper object of scientific knowledge" (ibid, 14).

According to Massimi, the crucial, distinctively new feature that Kant introduced in the conception of phenomena is "that a physical phenomenon - intended as a conceptually determined appearance - has built in it from the very outset the concept of a moving force as the cause of the observed appearance. It is the causal concept of a moving force that distinguishes phenomena from appearances, or better, that transforms appearances into phenomena, i.e., objects of possible experience into objects of experience" (ibid, 14). I agree with Massimi that a Kantian conception of phenomena does better justice to the complexity of phenomena in scientific practice - in particular, by stressing that (representations of) phenomena entail conceptual content introduced by how humans (cognitive agents) conceptualize perceptions and experiences. But new issues emerge that I will address shortly.

\subsection{What does 'representation of a phenomenon' mean?}

Based on this brief overview of ideas about phenomena, another problem emerges, because it has become unclear whether the word 'phenomenon' refers to a real-world thing, say a physical phenomenon 'out there,' or instead, to something that might just as well be called a representation. The notions of observable and non-observable phenomena in philosophical 
literature on scientific models suggest that phenomena are real physical things that we can look at or point to. But Massimi's Kantian account of phenomena assumes that phenomena are conceptually determined appearances. This seems to suggest an idealism that I would rather avoid here. Therefore, I propose firstly, that we must introduce the concept of representations of phenomena to give phenomena a proper place between data and models or theories, and also, to make the phenomenon suitable for epistemic activities such as modelling them. Secondly, close to Massimi, my view is that the representation of a phenomenon involves data being transformed into epistemic entities through mathematical, theoretical and conceptual frameworks.

The progress made in this way is that our representations of phenomena can no longer be understood as if phenomena are somehow 'literally' read off or depicted from reality, but must be understood as representations that are the result of an interplay between our experiences, observations and data (the 'appearances'), on the one hand, and mathematical, theoretical and conceptual frameworks on the other. In Section 6 it will be worked out somewhat further that the role of these frameworks must be interpreted as perspectives.

As a consequence, notions such as 'descriptions of (unobservable) phenomena' and 'models as representations of (unobservable) phenomena' cannot be intuitively grasped by picture-like views of phenomena. The account of phenomena presented here shows that mathematical, theoretical and conceptual content is built in representations of phenomena. Subsequently, it is precisely this content that enables forms of inferential reasoning by scientific researchers that go beyond mere deductive or inductive forms of reasoning. In this way, we obtain a more adequate account of the role of phenomena in scientific practices. ${ }^{16}$

The conceptual distinction between phenomena and models has become blurred due to the distinction that must be made between the target-phenomenon 'that exist out there' and appear to us on the one hand, and 'descriptions or representations of phenomena' that count as epistemic entities, on the other. In the account proposed here, the latter should be understood as a 'conceptually determined appearance' rather than a 'literal image of the appearance.' Consequently, the 'description or representation of a target-phenomenon' is already a scientific model $_{1}$ of the phenomenon. Next, this 'description or representation of the target-phenomenon' (i.e., scientific model $_{1}$ ) may ask for an explanation. This will produce yet another scientific model $_{2}$ of the same target-phenomenon 'out there.' A more precise phrasing of the latter would say that 'the scientific model $_{2}$ represents an explanation of the target-phenomenon., ${ }^{, 17}$

\footnotetext{
${ }^{16}$ The concept of representations of phenomena presented here is close to accounts of scientific concepts by Nersessian (2009c), Feest (2010), and Boon (2012). The crux is that the mathematical, theoretical and conceptual content that is built in the conception of phenomena (although based on 'raw' data) is partly hypothetical and fictional (e.g., Vaihinger 1911), rather than fully empirically grounded (as in the semantic view). Nonetheless, the resulting representation must meet pragmatic and epistemic criteria such as internal coherence and logical consistence, intelligibility in the sense of physical conceivability (Massimi forthcoming), physical plausibility, and empirical adequacy (Van Fraassen 1980). Additionally, it is precisely thanks to the conceptually meaningful but still hypothetical and fictional content built into the representation of the phenomenon that different kinds of inferential reasoning are made possible. Outcomes of inferential reasoning based on the representation of the purported real-world non-observable phenomenon can be tested against reality. In this manner, it is tested whether the hypothetical content and what this content allows to infer from it meets epistemic criteria such as empirical adequacy.

${ }^{17}$ Clearly, the first and the second model are not identical. Further analysis could aim to better understand their semantic and epistemological relationships. This issue will not be elaborated here.

${ }^{18}$ Also see Bokulich (2009), who sees the explanatory power of models as being closely related to their fictional nature.
} 


\section{The construction of models as part of an epistemology of scientific models}

\subsection{Philosophical issues in an epistemology of scientific models}

The discussion so far makes plausible that an epistemology of scientific models must address a number of philosophical issues that arise when we let go of the picture-metaphor of models to explain how scientific models represent non-observable target-phenomena. These concern respectively: (a) Alternatives to the picture-metaphor of scientific models; (b) The assumption that scientific models are mere non-linguistic entities; (c) The question what a real-world targetphenomenon is; (d) The issue of representing non-observable real-world phenomena; (e) The justification of models; (f) The question how models allow for inferential reasoning about the realworld target-phenomenon. These issues seem non-existent as long as the picture-metaphor of models is maintained. However, this metaphor, in which the model is a more or less literal picture of the real-world target-phenomenon, appears problematic as the intuitive basis for our understanding of scientific models. This implies that an epistemology of models must provide an alternative to 'how models represent' in a way that satisfactorily resolves these issues. Here, I propose an alternative that does not take the ready-made model and target-phenomenon as the point of departure, but starts from the question of how scientific models are constructed - as this should clarify the questions raised in the transcendental and pragmatic approach aimed at an epistemology of models that suits scientific practice, such as: What are scientific models? What do scientific models represent exactly? How are scientific models connected to the real world? How is it possible that scientific models enable epistemic tasks related to real-world target-phenomena?

Below, the implications of taking the model construction into account in an epistemology of scientific models will be discussed along the lines of the issues (a-f). To this end, I will use a systematic account of the (re)construction of models as proposed by Boon and Knuuttila (2009) and Knuuttila and Boon (2011), which has been elaborated into a concrete, practically useful method, called the B\&K method for the (re)construction of scientific models in scientific practices (Boon 2020). ${ }^{19}$

\footnotetext{
${ }^{19}$ The proposed B\&K method consists of ten questions to systematically determine the concrete aspects that are built into the scientific model (Boon 2020). This list can be employed to construct a model but also to reconstruct how an existing model was put together. In short these questions are: What is/are the:
}

1. Problem context (which may refer to the socio-technological problem)?

2. Target-system or physical-technological phenomenon $(\mathrm{P})$ for which the model is constructed?

3. Intended epistemic function(s) of the model? (which refers to inferential reasoning in regard to the problem stated in aspect 1).

4. Model type? (for example, a causal mechanist, or a mathematical model; this choice is related to the intended epistemic function).

5. Relevant (physical and/or technical) circumstances and properties (e.g., by which variables is a non-observable phenomenon connected to the tangible world, or, by which variables is the phenomenon or target-system affected)?

6. Measurable (physical-technological) variables (i.e., how is the phenomenon identified or connected to the tangible world)?

7. Idealizations, simplifications and abstractions (e.g., concerning aspects 2, 5 and 8)?

8. Knowledge used in the construction of the model (e.g., theoretical principles and knowledge, knowledge of sub-phenomena, phenomenological laws, empirical knowledge)?

9. Hypotheses (e.g., new concepts and explanations) built into the model?

10. Justification or testing of the model? (Also see Section 5, issue e). 


\subsection{Issue a: Alternatives to the picture-metaphor of scientific models - Models as hubs}

By starting with the question of how models are constructed, it becomes clear that models are rather a kind of $h u b s$ where heterogeneous aspects are brought together and merged into a coherent whole (cf. Nersessian and Patton 2009; Nersessian 2009a, b), which is then called the scientific model of a target-phenomenon. This alternative take on models shows that such an intricate construction process in which different types of content are chosen and merged, most probably does not result into a more or less literal picture of the targetphenomenon. An account of models as constructed epistemic entities (rather than being logically or algorithmically deduced from abstract theories, as in the semantic view), therefore, overcomes the idea that scientific models, metaphorically speaking, are more or less literal (in the sense of structurally similar) pictures of their target.

\subsection{Issue b: Are models mere non-linguistic entities?}

A widespread, although somewhat implicit, assumption is that models are non-linguistic entities, which may be a relic of the semantic view. In Section 2 it was explained that the advantage of this assumption is that semantic notions such as isomorphism or similarity can account for the representational relationship between observable structures or patterns. However, as was argued in Section 3, the idea of scientific models as mere non-linguistic entities is too limited. When we consider how actual scientific models of real-world phenomena are constructed, it is clear that models cannot be derived from abstract theories alone, nor can they be generated by a direct picture of reality. Instead, modeling is a construction process in which heterogeneous content is collected and combined into a coherent whole. When we look at the aspects that are built into the model (as in note 19) and think of models that are presented in the scientific literature, it becomes obvious that models are rather a kind of story about the phenomenon. In scientific articles, this story is usually clarified with pictures, diagrams and graphs. But the idea that these non-linguistic elements are 'the model' is mistaken. We should rather adopt the idea that the model consists of the entire content of a scientific article.

\subsection{Issue c: What is a real-world target-phenomenon?}

The question of characterizing the target-phenomenon represented in the model is usually not addressed in the literature of models. ${ }^{20}$ Knuuttila and Boon (2011), on the other hand, claim that developing a conception of the real-world target-phenomenon is an inherent part of the modelling process (note 19, aspect 2). Therefore, the 'description' of the target-phenomenon, and the scientific model of the

\footnotetext{
${ }^{20}$ A notable exception is Contessa (2010), who discusses the ontological status of models, thus distinguishing between three types of models (material, mathematical, and fictional). Contessa argues that the ideal pendulum described in physics textbooks is not a material, nor a mathematical but rather a fictional object. Accordingly, Contessa argues that fictional models represent fictional entities. My notion of imaginary or imagined phenomena appears close to Contessa's notion of fictional objects. However, although Contessa raises the question of how the model refers to the real world, he does not provide a satisfactory account of the semantic and epistemic relationships between fictional entities (which in his view are imaginary objects, such as the ideal pendulum) and real-world objects. Also see Toon (2012).
} 
target-phenomenon are co-constructed epistemic entities. ${ }^{21}$ An example is how Sadi Carnot constructed the model of the ideal heat engine. ${ }^{22}$ By abstracting from much of what seems to characterize real-world heat-engines, Carnot conceives of heat-engines in terms of a phenomenon described as 'heat is converted into motivepower.' This is the imagined phenomenon for which he then constructs the model. In this way, imagining (i.e., constructing a 'description' of) the target-phenomenon is part of the modelling process. ${ }^{23}$

In line with Massimi's (2008) Kantian account of phenomena discussed above (Section 4), this example shows that the description of the target-phenomenon is not 'ready-made.' It is not an empirical manifestation of what there is, but instead, an interpretation of an observed or experienced object, property or process such as the real heat engine as experienced and interpreted by Carnot. Such an interpretation requires to bring observations, experiences, or data under (scientific) concepts (i.e., 'subsumption under concepts'). ${ }^{24}$ This can be concepts that already exist, or newly invented scientific concepts (Feest 2010; Boon 2012). Crucially, in this way conceptual content enters into the model.

Additionally, the way in which target-phenomena are conceived in scientific practice involves the kind of practical and/or scientific problem that the researcher aims to tackle (note 19, aspect 1). In the example of Carnot this implies that his conception $^{25}$ of the phenomenon (the imagined phenomenon) not only

\footnotetext{
${ }^{21}$ The claim that the construction of a model first requires a representation of the target-phenomenon seems confusing with regard of the general idea that models are representations of their target-phenomenon. Two responses are possible. Firstly, the representation of the target-phenomenon can indeed already serve as a preliminary model (which is an idea proposed in Knuuttila and Boon 2011). Secondly, as already indicated in Section 4, scientific models are often more than a strict (e.g., 'literal,' 'picture-like,' or 'descriptive') representation of the phenomenon, because models usually offer explanatory or theoretical (e.g., mathematical) interpretations of the target-phenomenon. In this case, the phrase "the model represents the targetphenomenon" actually means that the model represents an explanation of the phenomenon. Pushing this further, it can also be said that "the model represents the phenomenon 2 (e.g., a causal mechanism) that explains the target-phenomenon ${ }_{1}$."

${ }^{22}$ Carnot's construction of the model of the ideal heat engine is a case that cannot be grasped by the original semantic view of theories, because the model is not derived from an abstract theory. Instead, thermodynamic theory emerged from Carnot's model of the ideal heat engine. Unfortunately, many textbooks in thermodynamics present the ideal heat engine as if it derives from thermodynamic theory, that is, as if thermodynamic theory made the invention of heat engines possible.

${ }^{23}$ Frigg and Nguyen (2016a), in their DEKI account of representation, use Kendrew's plasticine model of myoglobin as an example. In this example, myoglobin is the target-system T. Hence, in my vocabulary, myoglobin, is the purported non-observable real-world target-phenomenon that is represented in the model. According to Frigg \& Nguygen, the model M denotes its target system T, and denotation is the core of representation. However, although their DEKI account of representation claims that denotation of the targetsystem is crucial to modelling, they do not explain how the target system $\mathrm{T}$ is denoted, i.e., how it is possible that scientific researchers denote, identify, indicate, or whatever you call it, a target-phenomenon. This problem is at the core of my article.

${ }^{24}$ Vaihinger (1911), in a Kantian fashion, stressed the importance of subsumption under concepts, which is a crucial part of his notion of 'as if' reasoning in scientific practice. By bringing observations or experiences under a concept an imaginary phenomenon is generated (e.g., a regularity, a law, an invisible entity, or a property). Subsequently, the structure or content of the concept enables epistemic agents to reason about that imaginary phenomenon - i.e., it enables inferential reasoning through the structure and content introduced by the concept. It is important to see that in this manner, Vaihinger defends an anti-realist position in the sense that subsumption under concepts enables 'as if' rather than 'it is' reasoning.

${ }^{25}$ My use of the words 'conception' or 'conceptualize' is similar to Rouse's (2011) notion of 'conceptual articulation.' I use the two notions interchangeably.
} 
encompasses abstract or theoretical concepts such as 'heat,' 'motive power,' and 'conversion,' but also practical or functional concepts concerning the practical problem he aims to solve by his theoretical approach. ${ }^{26}$

In scientific practice, therefore, determining and characterizing the real-world targetphenomenon already involves theoretical and conceptual interpretations of data and experiences as an inherent part of the model construction. As a consequence, the 'description' of the real-world target-phenomenon is the imaginary phenomenon. Furthermore, the target-phenomenon is detected (in the real world) by the same measurable variables that characterize the imaginary phenomenon (in the model), securing a representational relationship between a structure generated by the model and a structure generated by the real-world target-system (as in the semantic view).

\subsection{Issue d: How do models represent non-observable real-world target-phenomena?}

Characterizing the semantic and epistemic relationship between the model and the target-phenomenon in terms of similarity, likeness, etc., makes sense in a picturemetaphor of representation. However, when the representational relationship concerns purported non-observable phenomena it is difficult to understand what 'similarity' means, because a direct visual comparison no longer seems to have a clear meaning. Alternatively, the possibility to connect (i.e., to draw a cogent semantic relationship) between the model and the real-world is warranted by how the model is constructed. In particular, when researchers choose the variables that characterize or causally affect the (observable, or the purported non-observable) target-phenomenon, they are guided by which variables are detectable or measurable (see note 19, aspect 6). In other words, these variables are not randomly chosen, nor do they emerge from nowhere. Note that this emphasis on the role of measurable data in constructing scientific models of realworld phenomena complies with how connections between theory and world are established according to the semantic view (Section 2). Thus, by including the relevant measurable (physical-technological) variables in the construction of a scientific model, a connection is warranted between the variables that characterize the imaginary phenomenon in the model and those that characterize the real-world targetphenomenon (also see issue c).

\subsection{Issue e: The justification of models}

The question of how models are justified is not generally dealt with in the literature on models. This may be due to the idea that similarity between model and targetphenomenon suffices as justification: either objectively determined by the comparison between the structure of the model and that of the target, as in the semantic view, or

\footnotetext{
${ }^{26}$ The practical problem is improving the 'useful effect' (which at some point got translated into 'energyefficiency') of real heat engines. Carnot translates this into a theoretical problem as follows: "The question whether the motive power of heat [i.e. the useful effect that a heat engine is capable of producing] is limited or whether it is boundless has been frequently discussed. Can we set a limit to the improvement of the heatengine, a limit which, by the very nature of the things, cannot in any way be surpassed? Or conversely, is it possible for the process of improvement to go on indefinitely?" [Sadi Carnot (1824), Reflexions on the Motive Power of Fire and on Engines fitted to develop that Power].
} 
subjectively determined by researchers, as in the accounts by Giere and Suárez. Instead, Knuuttila and Boon (2011) emphasize that models are partly justified by how the model is built. This involves a creative process of critically searching, selecting, conceptualizing, assessing and combining supposedly adequate and relevant heterogeneous aspects that are forged into a coherent whole (note 19). One of these aspects is the choice of relevant and measurable variables to characterize both the imagined phenomenon represented in the model and the purported real-world phenomena. Another one is the choice of scientific concepts to characterize the phenomenon.

Scientific models are justified and tested in at least three ways that complement each other, namely: (i) by justifying the relevance, physical plausibility, and adequacy of aspects that are built into the model; (ii) by assessing whether the model meets relevant epistemic and pragmatic criteria such as internal coherence, internal consistence, intelligibility and physical plausibility, ${ }^{27}$ and adequacy with regard to the current state of knowledge; and (iii) through empirical or experimental testing against reality by comparing model-outcomes and experimental results in order to achieve empirical adequacy. ${ }^{28}$

In the second part of this article (Section 6), I will argue that the choices of the heterogeneous aspects are also guided and justified by the disciplinary perspective within which the researcher works.

\subsection{Issue f: How do scientific models allow for inferential reasoning?}

An important aspect of an epistemology of scientific models is to explain how models can be sources of (new) knowledge. To be useful for researchers in performing epistemic tasks, scientific models must enable inferential reasoning, either internal to the model in regard of the imaginary object represented in the model (as is emphasized in fictional accounts of models, e.g., Barberousse and Ludwig 2008; Suárez 2009a, b; Contessa 2010, Toon 2012), or externally oriented to generate model-outcomes that describe or represent aspects of the real-world target-phenomenon.

Suárez $(2003,2004)$ proposes an inferential conception of representation, which entails the idea that "[the internal structure of the representation] A allows competent and informed agents to [correctly] draw specific inferences regarding [the target] B" (Suárez 2004, 773). This does not require "that A [merely] allows deductive reasoning and inference; any type of reasoning - inductive, analogical, abductive - is in principle allowed, and A may be anything as long as it is the vehicle of the reasoning that leads an agent to draw inferences regarding B" (ibid, 773). Furthermore, Suárez stresses that 'correctly drawing inferences' is not equivalent to 'drawing inferences to true conclusions.' I adopt Suárez's idea that scientific models allow inferential reasoning by researchers, and also, that inferential reasoning can be any type of cogent reasoning.

\footnotetext{
${ }^{27}$ These epistemic criteria (intelligibility and physical plausibility) seem to resonate with Massimi's (forthcoming) notion of physical conceivability.

${ }^{28}$ Note that the semantic notions isomorphism and similarity to describe the semantic relationship between model and world only applies to this third way of testing a scientific model, that is, to the semantic relationship between the model-outcome and the experimental-outcomes. This accords with ideas about testing against reality in the semantic view. However, this is only one part of the testing of a model. It is important to recognize that the other two ways of testing are not through somehow assessing isomorphism or similarity between model and world.
} 
However, unlike Suárez's deflationary notion of representation, I argue that an epistemology of scientific models must also explain how it is possible that models enable inferential reasoning, ${ }^{13}$ not only internally, but also related to (purported nonobservable) real-world target-phenomena. ${ }^{29,30}$ First of all, as scientific models are not, or not exclusively derived from abstract theories, it must be explained how models are constructed and justified that make these kinds of epistemic activities possible at all, for which the B\&K method is proposed (note 19). Next, based on the analysis so far, I assume that: (i) the full content of the model - consisting of the 'coherent story' constructed by integrating the aspects put forward in the B\&K method (note 19) - allows for such reasoning; (ii) therefore, not only the (non-linguistic) internal structure of scientific models allows for different types of inferential reasoning about the target-phenomenon, but also the conceptual content and other aspects built into the model, such as knowledge regarding the physico-technological interactions with the (supposed) real-world target-phenomenon and theoretical knowledge; and (iii) as has been argued in Section 2, 'direct' comparison between model (model-outcomes) and an unobservable target-phenomenon is (only) possible by means of the measurable or detectable features by which the phenomenon is characterized (also see issue e). In the second part of this article (Section 6), I will argue that the disciplinary perspective is the broader framework through which the construction, justification and reasoning with models is possible.

\subsection{Taking stock: Towards an epistemology of scientific models}

In the first part of this article, I have argued that it needs to be clarified what exactly we mean when we talk about scientific models that supposedly represent non-observable phenomena. Getting a philosophical grip on this is relevant for better understanding scientific practices. The intuitively plausible idea about scientific representation, expressed in sentences such as "[S]cience provides us with representations of atoms, ..., and the world's climate" (Frigg and Nguyen 2016a), and, "scientific models represent their target-phenomenon," is problematic. On the basis of my analysis so

\footnotetext{
${ }^{29}$ Philosophers may disagree whether this question is worth philosophical analysis. Giere, for example, rhetorically asks: "Do we, as theorists of science, need to give a more detailed account of the processes of interpretation..? I think not. We can pass this job off to linguists and cognitive scientists. We know it can be done because it is done" (Giere 2010, 271). Similarly, Suárez (2012), in his review of Bailer-Jones' monograph, denies that her "burning question" deserved explanation. Her burning question is: "How is it that there is something about the model that allows us to demonstrate something that then, after appropriate interpretation, becomes applicable to and insightful about real-world phenomena?" (Bailer-Jones 2009, 197). Both Giere (2010) and Suárez (2004) thus shift the question of how it is possible that models allow for inferential reasoning to the competent and informed agent. Clearly, I disagree with them on this issue. With regard to scientific practices this is the fascinating and difficult to solve aspect of scientific representation. In particular, when models supposedly represent target-phenomena that cannot be observed in a direct and straightforward way (such as DNA), it is difficult to understand, even for competent researchers, how the scientific model is similar to its target-phenomenon or why its structure allows correct inferential reasoning about it. Their accounts, therefore, are not very informative about the epistemic functioning of models and modelling in scientific practice.

${ }^{30}$ Also see Toon (2012), who argues that the more sophisticated version of Giere's (2010) similarity view that appeals to the role played by scientists and their representational capacities is not yet sufficient. Instead, we must describe how it is that scientists use models to represent, and proponents of the similarity view "must offer a different account of how similarities are put to work in scientific representation" (ibid, 255).
} 
far an epistemology of scientific models that suits scientific practices can be summarized by the following statements:

(1) An epistemology of scientific models should not be guided by a picture-metaphor but take into account how scientific models are constructed.

(2) Scientific models are constructed by combining heterogeneous aspects (as in note 19), which researchers must integrate into a coherent whole that allows different kinds of inferential reasoning. Additionally, in the process of developing them, models are a kind of $h u b$ where these heterogeneous aspects are brought together.

(3) In order to be meaningful and intelligible for scientific researchers, scientific models must also contain linguistic (i.e., conceptually meaningful epistemic) content. This implies that scientific models tell a kind of story rather than being self-explanatory pictures. For that reason, it is better to assume that the full content of a scientific article about a specific target-phenomenon is the scientific model. Indeed, the story told in scientific articles is clarified by means of mathematical formula, graphs, diagrams and pictures, but the idea that these non-linguistic elements are the model is mistaken.

(4) Constructing a model of a target-phenomenon requires a representation (or 'description') of the real-world target-phenomenon to begin with. This representation is not 'ready-made' but is also the result of creative and constructive activities by researchers. As a consequence, the conceptual articulation of a target-phenomenon is usually part of the modelling process. ${ }^{31}$

(5) Scientific models are tested and justified in at least three different ways that complement each other.

(6) Scientific models enable inferential reasoning (and more broadly, epistemic activities) through the entire content built into the model, which encompasses the (nonlinguistic) internal structure, the conceptual content (e.g., scientific concepts), the physico-technological context, theoretical knowledge, etc. (see note 19).

(7) Scientific models are constructed within a specific scientific discipline. Within this discipline, epistemic activities, such as conceptualizing target-phenomena and constructing scientific models, are guided and constrained by a disciplinary perspective. This is the topic of the second part of this article (Section 6).

\section{Disciplinary perspectives in the construction of scientific models}

\subsection{Disciplinary perspectives in science}

My philosophical argument for defending the indispensable role of disciplinary perspectives in science can be summarized as follows. First, the finding that a direct 'picture-like' representational relationship between scientific models and targetphenomena is problematic for philosophical reasons, leads to the idea that this

\footnotetext{
${ }^{31}$ The idea that the conceptual articulation (and representation) of the target-phenomenon is an inherent part of the model, to some extent corresponds to ideas that are put forward in a fiction-view of models. However, my worry is that fiction views of models do not explain the semantic and epistemic relationship between the imaginary phenomenon represented in the model and the real-world target-phenomenon.
} 
representational relationship must be clarified by investigating how scientific models are constructed. Subsequently, I argued that scientific models in scientific practices are not mere non-linguistic entities (e.g., observed patterns, or mathematical structures derived from abstract theory), but are better understood as hubs in which heterogeneous aspects are combined into a coherent whole that consists of both linguistic and nonlinguistic content. Also, the construction of models (as well as the conceptual articulation of the phenomena) requires creative and constructive epistemic activities by researchers, who search, assess, choose and integrate the heterogeneous aspects that they built into the model. ${ }^{32}$ This involves epistemic activities such as, to select, organize, structure and interpret empirical data or phenomena by choosing (or inventing) relevant epistemic components. Examples are: fundamental principles; mathematical structures; physically meaningful concepts (e.g., elasticity, oscillation, force); practical and theoretical knowledge (e.g., abstract theory; phenomenological and scientific laws); and, explanatory hypotheses. In turn, epistemic activities are guided by epistemic and pragmatic criteria such as, coherence, consistency, adequacy, intelligibility, physical plausibility and relevance. Finally, I suggest that the epistemic activities, as well as the choices and judgments that are needed in the construction of scientific models are guided and enabled by the disciplinary perspectives of the practice within which the researcher works.

\subsection{The roles of scientific and instrumental perspectives in scientific practices}

Metaphorically, perspectives are like a pair of glasses. They enable to see aspects of the world in a specific way. The strong version, which I endorse, holds that without perspectives nothing meaningful is seen or known. Perspectives in this strong version are more like a pair of eyes. Perspectives are therefore not representations, but the means by which representations are generated. Giere (2006) and Van Fraassen (2008) have argued that different types of perspectives feature in science, enabling different kinds of both instrumental (technological) and epistemic activities to generate representations. Based on their insights I distinguish different kinds of perspectives in scientific practices:

Firstly, it is only through scientific perspectives in the sense of mathematical and/or conceptual frameworks (including laws, scientific concepts and phenomenological analogies) and fundamental (ontological) beliefs that researchers 'recognize' a realworld target-phenomenon in 'raw' observations, experiences and data. It is only possible, therefore, to form a mathematically or conceptually meaningful representation of a phenomenon out of 'raw' data by means of these kinds of perspectives. Without them, no phenomenon is seen or recognized (also see Rueger 2005).

Secondly, researchers generate 'raw' data using (technological) instruments and experimental procedures, which therefore count as instrumental perspectives (Giere 2006; Van Fraassen 2008). Based on the analysis of the semantic view in Section 2, I add that the data is therefore of a specific type, namely, as determined by the instrumental perspective. Thus, the instrumental perspective determines the specific way in which the purported phenomenon is characterized and represented. For

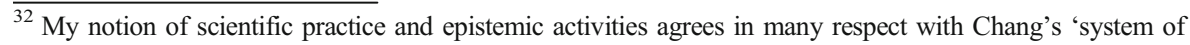
practice' $(2014,2020)$.
} 
example, the behavior of a bullet is conceptualized as a trajectory, and represented by the measured variable, i.e., the $x-y-z$ location as a function of time, and not the mass, shape or roughness. ${ }^{33}$

Thirdly, researchers use practical and theoretical knowledge about the instruments and procedures indicative of a purported non-observable real-world phenomenon to predict the occurrence of this phenomenon elsewhere (cf., Boon 2017). The crux is that although researchers do not have direct access, they suspect that a non-observable phenomenon must be present in the case of physical-technological conditions at which the (non-observable) phenomenon is believed to exist. This type of knowledge about instruments and procedures therefore offers a scientific and instrumental perspective on physical or technological systems, with which researchers can produce knowledge about already known but unobservable aspects of that system.

Fourth, similar to the conceptual articulation of phenomena, the construction of scientific models requires that researchers use theoretical, mathematical and conceptual frameworks to structure, interpret or explain the real-world target-phenomenon (note 19 , aspects 8 and 9). In this way, structure and conceptually meaningful content is built into the model, which is crucial for becoming a representation at all. Giere (2006) argues that the used frameworks are scientific perspectives.

Fifth, epistemic entities such as 'representations of phenomena' and 'scientific models' can themselves acquire the role of scientific perspectives for performing epistemic tasks. Scientific models, for example, allow different kinds of inferential reasoning, such as deductive, inductive, predictive, explanatory, explorative, hypothetical and 'what if ...' reasoning about the target-phenomena. In this capacity, the model functions as a perspective with which new hypotheses, knowledge or questions regarding the target-phenomenon are generated. ${ }^{34}$ In addition, scientific models as perspectives also make inferential reasoning possible about interventions with physical or technological systems. For example about how you could manipulate, control, generate or create the (purported) real-world target-phenomenon.

This list shows different types of perspectives and their roles in scientific practices. Below, I will propose a more systematic (Kuhnian) framework to characterize disciplinary perspectives.

\subsection{The role of disciplinary perspectives in constructing a phenomenon}

The construction of ('descriptions' or representations of) target-phenomena takes place in a broader disciplinary context. I propose to call the intellectual, epistemic and conceptual part of the discipline the disciplinary perspective of a scientific practice. Briefly said, the disciplinary perspective enables researchers in a specific scientific discipline to reason and conceptualize, but it also constrains them. Therefore, 'conceiving of' (or, 'conceptually articulating') the targetphenomenon does not take place in void, but already involves many different aspects that are known to, and understood by the researcher. More specifically, the imagined phenomenon is

\footnotetext{
${ }^{33}$ According to Giere (2006), this phenomenon is then interpreted as a Newtonian system. Hence, Newton's theory is used as a theoretical perspective to generate a scientific model of the phenomenon, i.e., a representation that interprets or structures the phenomenon in a very specific way. Vaihinger (1911) would rather say that the phenomenon is subsumed under Newton's laws of motion. The resulting scientific model then allows 'as if' reasoning about the phenomenon.

34 Boon and Knuuttila (2009) and Knuuttila and Boon (2011) call models epistemic tools. In fact, they thereby point at this capacity of scientific models to be used (in the current vocabulary) as perspectives in epistemic tasks.
} 
embedded in a network consisting of different types of intellectual, epistemic and conceptual aspects. Examples are: partially interpreted (i.e., conceptualized) real-world experiences (e.g., the workings of heat, motive-power and heat-engines); scientific concepts and conceptual frameworks (e.g., 'caloric,' 'heat,' 'motive-power,' etc.); established practical knowledge (e.g., about the workings of experimental set-ups); empirical and phenomenological knowledge (e.g., about experimental findings by other scientists); fundamental principles (e.g., heat cannot flow from cold to hot); theoretical knowledge (e.g., the gas-laws of Boyle and others); measurable variables (e.g., mass, volume, pressure, and temperature); and, established conceptually meaningful parameters (e.g., 'density,' 'specific heat'). ${ }^{35}$ A closer look at these aspects makes it clear that this network connects the imagined phenomenon with both the real-world target-phenomenon (e.g., in terms of measurable variables) and with mathematical and conceptually meaningful content (e.g., through theories and concepts). ${ }^{36}$

The network of aspects through which a phenomenon is identified is an important part of the disciplinary perspective that guides and also constrains (communities of) researchers. It enables them to perform creative, critical and constructive epistemic activities in the construction of representations and in the use of models for inferential reasoning. Accordingly, the question of how the representational relationship between the imagined and the purported real-world target-phenomenon is to be understood is explained by how they are embedded in the disciplinary perspective. On the one hand, the imagined phenomenon in the model is conceptualized within the disciplinary perspective. On the other hand, the real-world phenomenon is characterized or determined by experimental setups or (technological) measurement procedures typical of the discipline, and accordingly represented in a specific way through the disciplinary perspective. More specifically, the specific constellation that characterizes or determines the purported non-observable real-world phenomenon includes reference to relevant measurable variables and parameters, measuring instruments and experimental set-ups, along with theories, concepts and models that describe or explain these instruments and experiments, and, depending on where one starts the research, reference to the imagined phenomenon. ${ }^{37}$

\footnotetext{
${ }^{35}$ These examples of relevant aspects form the disciplinary perspective within which Carnot conceptualized the phenomenon ("heat is converted into motive-power") and constructed the model of the ideal heat engine. They can be found in Carnot's (1824) treatise.

${ }^{36}$ One of the reasons for endorsing perspectivism in the philosophical literature, is the concern that scientific practices use multiple conflicting models to explain and understand the same phenomenon (e.g., Rice 2020; Fagan 2020; Mitchell 2020). However, when accepting my suggestion that unobservable phenomena are defined and characterized within a complex constellation of different aspects, authors could look more critically at what "the same phenomenon" means. Wolff (2020) provides a comprehensive point of departure for such an investigation. 'Alcohol addiction' is my own simple example to show that 'the same phenomenon' may be a problematic notion, because this 'observable' phenomenon is turned into a much more sophisticated conception when studied in either sociology, psychology, neurobiology, or genetics. In each of these scientific disciplines, the conception of the phenomenon is linked to theoretical concepts and measurement procedures of that discipline, as well as to the specific research questions asked in the discipline, usually in view of the practical (societal) problem. Therefore, it is not at all 'the same phenomenon' that is modelled in different disciplines.

${ }^{37}$ In several respects my view of phenomena corresponds with Rouse's $(2009,2011)$ ideas about the roles of scientific concepts and hypothetical entities in scientific practice. According to Rouse, laboratory work and experiments play a crucial role in articulating and consolidating conceptual understanding. He stresses that experimentation is integral to conceptual articulation of the phenomenon. This also involves the idea that activity and practice precede ontology, an idea that I endorse in the present article. Accordingly, in my terminology, phenomena are postulated in the interaction between experimenting, measuring and conceptualizing. It is through the intellectual activities of researchers that a (non-observable) real-world phenomenon is conceptually postulated, while at the same time the purported phenomenon is physically determined, characterized and established by the researchers' practical activities (e.g., experimenting and measuring).
} 
In sum, instead of a picture-like representational relationship between the imagined and the purported real-world target-phenomenon, both are conceptualized and identified through specific constellations within the discipline. This makes their representational relationship much more complex. At the same time, the conceptually rich representations of both imagined and real-world target-phenomena that are crafted with the help of a disciplinary perspective enable different kinds of inferential reasoning by researchers, leading to new creative ideas, relevant questions and challenging hypotheses.

\subsection{A Kuhnian framework for characterizing disciplinary perspectives}

As was suggested above, researchers in specialized fields or disciplines have developed and internalized perspectives that give direction to how they approach research, which is called a disciplinary perspective. Researchers have adopted the disciplinary perspective, often without being fully aware of it, since the perspective is not usually explicitly conveyed or reflected upon. A metaphor for this role of the disciplinary perspective in becoming a researcher is that they have learned to look through a specific kind of spectacles, which they now wear without noticing it. In a Kuhnian fashion, I suggest that disciplinary perspectives can be characterized as consisting of heterogeneous but mutually cohering elements that support and reinforce each other. These aspects are listed here:

(i) Intrinsic aims and objectives related to what is considered the subject-matter of research in the discipline, usually reflected in the name of the discipline. For example, mechanics, chemistry, systems biology.

(ii) Practical purposes that are related to ideas about the extrinsic, practical relevance of the research-projects in the discipline. This purpose is usually reflected in the name of applied scientific fields, such as in technology (e.g., membrane technology), medicine (e.g., oncology, immunology), and agriculture (e.g., plant pathology). These disciplines raise research questions oriented at practical applications such as: "Can we improve the energy efficiency of steam engines?" "Can we biomimic photosynthesis to harvests sunlight?" "Can we find a drug for this type of cancer?" "Can we find a cure for alcohol addiction?" "Can we cultivate a species that is resistant to this pest?"

(iii) Research questions typical of the discipline, which are related to the intrinsic and extrinsic aims of the discipline. For example: "What is the mechanism that explains these phenomena?" "Which genetic factors are related to alcohol addiction?" "Which factors in an individual's personal history contributes to alcohol addiction?" Also see Carnot's research question in note 26.

(iv) The types of real-world phenomena (observable and non-observable) typically investigated in the discipline. Usually, the observable phenomena are related to the kinds of phenomena described in the external, practical purposes or problems that the discipline aims to engage with (e.g., technological functions, illnesses, pests). The non-observable phenomena are related to the more fundamental ontology of the discipline. They are the kinds of things in terms of which the discipline aims to understand and intervene with the observable phenomena. For example: Chemistry studies phenomena related to molecules. Microbiology 
studies phenomena related to micro-organisms. Biochemistry studies phenomena related to biochemical pathways. Psychology studies phenomena related to (the behaviour of) individuals. In this article, I have suggested that non-observable phenomena are characterized and defined by a specific constellation within the discipline. This includes for example: the data and observable phenomena indicative of the non-observable phenomenon; the measurement instruments and procedures that produce these data and observable phenomena; experimental set ups by which the purported phenomenon is investigated; and, the scientific concept or phenomenological law 'describing' it (also see Feest 2010; Boon 2012).

(v) Fundamental (ontological) principles, basic assumptions and beliefs used in the construction of scientific models and the conceptual articulation of non-observa ble phenomena. For example, fundamental principles in chemistry are conserva tion principles such as the principles that mass, atoms, charge and energy cannot appear or disappear.

(vi) Mathematical frameworks and axiomatic systems typical of the discipline. In my explanation of the semantic view, I suggested that mathematical equations entail variables and parameters that must be measurable in the discipline. For example, disciplines that make use of thermodynamics in their modelling of phenomena (e.g., when investigating aspects of 'artificial photosynthesis'), will also need to have measurement procedures to determine the variables and parameters in the mathematical equations that represent the thermodynamic properties of these phenomena (e.g., voltage, wave-length, thermal and electrical energy). Therefore, the (sets of) equations produced through these frameworks are interconnected with measurements and instruments specified in aspect viii below.

(vii) Theoretical (conceptual) frameworks and empirical (phenomenological) knowledge accepted in the discipline, including specific scientific concepts indicating observable and non-observable phenomena, and other technical terms. For example, chemistry uses scientific concepts that refer to perceivable properties such as 'colour,' 'acidity,' 'viscosity,' 'fluidity,' and 'crystallinity,' and also to purported non-observable phenomena such as 'atoms,' 'molecules' and 'chemical reactions.' The conceptual framework also encompasses theories and models that represent aspects of these phenomena, such as causal mechanisms that are held responsible for specific properties or chemical conversions.

(viii) Measurement instruments and procedures used in the discipline, including practical and theoretical knowledge about these techniques and procedures. For example, chemistry typically uses equipment such as a balance, a thermometer, a pH-meter, an Eh-meter, an oxygen-meter, a gas-flow meter, gas chromatography, and mass spectrometry. Researchers usually have practical and theoretical understanding of the workings of this equipment.

(ix) Research methods and typical strategies to investigate the phenomena. For example, disciplines usually develop specific types of experimental set-ups to investigate phenomena of interest (i.e., aspect viii). These are usually reported in the materials and methods section of a scientific article. Additionally, these methods and strategies are interconnected with and enabled by aspects v-vii above and aspects $\mathrm{x}$-xi below. 
(x) Epistemic and pragmatic criteria that epistemic results such as scientific models should meet. More general criteria that were already mentioned, are shared by most experimental practices. But also more specific criteria may apply, which have to do, for instance with the specific application context, such as 'reliability,' 'simplicity,' 'applicability,' 'specificity,' and 'predictive power.'

(xi) Representational means typical of the discipline, such as mathematical models, computer simulations, causal-mechanistic models, specific types of diagrams, pictures, and physical models.

The listed aspects i-xi together characterize the disciplinary perspective. ${ }^{38}$ Each of these aspects deserve further explanation. Also, their mutual relationships need to be worked out in more detail. Moreover, philosophers will undoubtedly wish to know how these aspects are justified, or whether they are necessary and sufficient. For the moment, my approach in listing is pragmatic, based on knowledge and understanding of existing research practices. Still some justification can be given. The reader may have noticed the overlap between this Kuhnian framework to analyse a disciplinary perspective, and the B\&K method for constructing or reconstructing scientific models (note 19). This should indeed be the case because researchers need guidance in drawing up a scientific model by making decisions and choices about the aspects listed in the B\&K method. The disciplinary perspective makes these decisions and choices possible. So, the disciplinary perspective guides and restricts the construction process. This means, therefore, that the elements indicated in the disciplinary perspective must cover the type of choices and decisions that are made when constructing scientific models.

Different scientific disciplines have different disciplinary perspectives, but I argue that each of them can be analyzed in terms of this Kuhnian framework (i.e., the aspects $\mathrm{i}$-xi). The concrete, discipline-specific disciplinary perspective is what researchers in that discipline 'have in mind.' Researchers are trained in using the disciplinary perspective. This gives them direction in their research efforts, for example in how to set up a research project and how to perform practical and epistemic activities in the discipline. Most often, the disciplinary perspective 'automatically' gives direction to how researchers conduct their research. But the disciplinary perspective does not function as an algorithm. Scientific research still involves a process of searching, choosing, and 'fitting together' (cf., Hacking 1992). Researchers must solve puzzles and make creative leaps to generate scientific models that meet the requirements of the discipline. And although the number of options is limited within a discipline, researchers still have to make numerous choices and decisions about what and how they conduct the research. In the types of choices that must be made, the aspects i-xi of the disciplinary perspective are guiding and constraining, although researchers are usually not explicitly aware of this.

\subsection{Evaluating disciplinary perspectives}

The claim in this article is that scientific models are constructed within the boundaries of a discipline and guided by that discipline's disciplinary perspective. This leads to an

\footnotetext{
$\overline{38}$ This list of elements (i-xi) is not meant to be exhaustive, nor will all these elements always be present or relevant when analysing and articulating a disciplinary perspective.
} 
(epistemological) anti-realism, such as defended by Van Fraassen $(1980,2008)$ and also Vaihinger (1911), which I endorse. The idea the disciplinary perspectives play an indispensable role suggests that each discipline can generate completely different and even incompatible models of reality. This consequence may give leeway to harmful forms of subjectivism and relativism. I contend that this can be avoided in different kinds of ways. Firstly, the construction process and the resulting scientific models must adhere to pragmatic and epistemic criteria that apply within the discipline (aspect $\mathrm{x}$ ). Secondly, several of the epistemic criteria operative in a scientific discipline transcend the specific disciplinary perspective. For example, internal coherence, logical consistence and empirical adequacy. Additionally, some of the theoretical frameworks transcend specific disciplines. Thirdly, the present article focuses on scientific practices that aim at scientific models (amongst other things) for adequately dealing with realworld problems (i.e., aspect ii). The focus on societally relevant epistemic purposes may allow for dealing in a more pragmatic fashion with controversies. Nevertheless, because of this societal purpose of scientific research, it is crucial that scientific results are critically evaluated with regard to epistemic and pragmatic criteria (i.e., aspect $\mathrm{x}$ ) that best fit the intended (societal) purpose. Fourth, I suggest that the disciplinary perspective can and should be analyzed and evaluated, for which this Kuhnian framework is proposed. Therefore, the disciplinary perspective can be critically examined, for example, by revealing erroneous (e.g., empirically testable) assumptions, logical inconsistencies, and incoherencies between elements (i.e., between the aspects i-xi). Also, shortcomings of methods or knowledge used in the discipline may be pointed out, which can result into improvements of specific aspects in this list. ${ }^{39}$ In this manner, by critically analyzing the disciplinary perspective and also learning from other disciplines, scientific disciplines can evolve and become enriched, refined, and/or (more) adequate for (additional) epistemic tasks.

\section{Overview and conclusions}

In Section 2, the semantic view of theories is interpreted as an epistemology of models. This leads to three important insights that come back throughout the article when explaining problematic aspects of scientific models.

The first insight is that, to understand 'how it is possible that models represent their target,' it is crucial to presuppose (as the semantic view does) that models are nonlinguistic entities, because this makes possible the comparison between models derived from abstract theories and data-models derived from experiments. ${ }^{40}$ Hence, due to the

\footnotetext{
${ }^{39}$ For example, an established discipline may incorporate new methodologies, mathematical frameworks, scientific concepts, theories and measurement techniques taken from other disciplines (Boon and Van Baalen 2019). An example of the transfer of methods, and the theoretical frameworks and measurement techniques that accompany these methods, is traditional scientific practices such as biochemistry that have evolved into biotechnology and systems biology. The traditional practice typically used experimental methods to produce causal-mechanistic models, but at some point, these practices adopted mathematical methods, which provided new opportunities, including mathematical models of the same system that allow for different epistemic uses. ${ }^{40}$ In the philosophy of science, scientific models are usually interpreted as non-linguistic entities, that is, picture, graphs, diagrams or 3D-objects that 'speak for themselves.' In short, non-linguistic entities consist of images (e.g., of a phenomenon in the real-world that people can perceive by observing it), whereas linguistic entities consist of descriptions (e.g., of a phenomenon in the real-world that people grasp by reading the text).
} 
fact that models, according to the semantic view, are (non-linguistic) structures it is possible to easily compare them and to decide whether they are similar in the sense of being (partly) isomorphic. From a scientific practice point of view, therefore, the representational relationship between the model and its target would be more or less unproblematic if models were mere non-linguistic entities.

The second insight is that the role of measurable variables is crucial for explaining how it is possible that a relationship can be established between a model and the real world. In the semantic view, the phenomenon that is imagined when deriving a model from the abstract theory is characterized in terms of measurable variables. In order to be comparable, the data-model that represents the real-world phenomenon must be characterized by the same measurable variables. This is how a semantic connection between them can be established. Otherwise, no comparison between them would be possible. Therefore, the representational relationship between the model and the targetphenomenon is based on representations in terms of measurable variables. Here as well, the representations being (non-linguistic) structures makes this relationship and comparison possible.

The third insight is that models derived from abstract theory, only represents the imagined phenomenon as a model-outcome, for example at (physical-technological) conditions in the experimental set-up. Therefore, models as 'direct' or 'literal' representations of (purported) non-observable phenomena is not an issue in the semantic view.

Section 3 discusses in what sense the notion of models as independent epistemic entities differs from the notion of models in the semantic view. Morgan and Morrison (eds. 1999) have argued that scientific models are not always derived from theories, but also have a 'life of their own.' That is why they regard models as 'autonomous epistemic entities,' which is a view that I accept as more suitable for scientific practice. But this implies that the semantic view, although cogent, provides a too limited view of the epistemic roles of models in scientific practice.

This requires rethinking how models represent. I explain why philosophical accounts that take scientific models as (more or less) literal representations prove to be problematic. This is particularly the case for models that represent purported (aspects of) real-world target-phenomena that cannot be perceived in a straightforward manner. ${ }^{41}$ Moreover, this also requires rethinking how it is possible to identify the phenomenon that is represented by the model, independent of that model. In addressing this, I conclude that an epistemology of scientific models also requires an epistemology of phenomena.

Section 4 explores ideas about phenomena in the philosophy of science in order to get a grip on the question how it is possible that scientific models represent nonobservable target-phenomena. Here, the key-issue is how we get to know the real-world phenomenon that is represented by the model. First, I refer back to the concept of phenomenon in the semantic view in which this concept seems unproblematic. In part, this is because phenomena are assumed non-linguistic observable entities (i.e., structures in terms of measurable variables).

\footnotetext{
${ }^{41}$ In this article, real-world target-phenomena that cannot be perceived in a straightforward manner are called 'purported non-observable real-world target-phenomena.' Focus is on physical or physical-technological realworld target-phenomena, but in some of the examples, I also refer to social phenomena.
} 
I then show that there are various interdependent issues that make both the concept of phenomenon, and the idea that models represent phenomena, rather blurry. Van Fraassen $(1980,2008)$ argues that phenomena are observable by definition, and denies unobservable phenomena. Bogen and Woodward (1988) aimed at a richer concept that agrees better to notions of phenomena in scientific practice. They assume that most phenomena are not observable in a straightforward manner. However, the distinction between data and phenomena proposed by them runs into trouble when they aim to avoid that phenomena are 'low-level' theories by assuming that phenomena are (derived from) patterns in data. As a consequence, their approach returns to the notion of phenomena in the semantic view. Still another idea about phenomena and their role in scientific practice is that phenomena are real-world things or occurrences that arouse our interest and ask for an explanation (Hacking 1983). In this view, scientific research starts with observed phenomena, rather than theories or data, and seeks to find explanations for these phenomena. This is an important addition, because research practices that target practical problems often start from thinking about phenomena. These can be phenomena observed in nature or produced in a laboratory. Moreover, the point of departure in a research project may also be non-observable phenomena that are postulated to explain observable phenomena. ${ }^{42}$

The issue raised by pointing out the different roles of phenomena in scientific practice is that scientific models represent the target-phenomenon not only in the sense of a ('literal') description or picture, but also in the sense of presenting an explanation for the phenomena. In the latter case, the scientific model that explains an observable target-phenomenon ${ }_{1}$ supposedly represents a non-observable phenomenon . $_{\text {. }}$

Based on this exploration of ideas about phenomena, it becomes obvious that phenomena, as objects of study in scientific practice, do not appear to researchers as 'ready-made' entities (Massimi 2007, 2008, 2011). Someone cannot simply point at a phenomenon and then photograph or draw or describe it. Instead, researchers inescapably use (theoretical) concepts to identify and conceptualize a phenomenon, usually within their own disciplinary perspective. The idea of 'scientific models as literal representations of target-phenomena' is therefore misleading. It wrongly suggests that researchers first point at a phenomenon, and then represent it - where this representation $i s$ the model. Altogether, I conclude that usually it is not possible to identify a phenomenon independent of any conceptual, theoretical or mathematical framework. This also implies that the identification of the target-phenomenon (be it 'observable' or 'non-observable') must be understood as an inherent aspect of modelling it (as explained in Section 5). Furthermore, the way in which that is done is guided and restricted by the disciplinary perspective (Section 6).

Section 5 argues that avoiding the picture-metaphor (i.e., the assumption of a similarity relationship between model and target) raises a number of philosophical

\footnotetext{
${ }^{42}$ Research could also aim at phenomena that do not even exist yet, but that are thought to serve some practical purpose. An example concerns the practical socio-technological problem of carbon dioxide emission in the production of electrical energy. An imagined solution is to harvest sun-light through artificial photosynthesis for the production of electrical energy. "Harvesting sun-light etc." and "artificial photosynthesis" are examples of phenomena of interest. So far, they are imagined phenomena, and scientific research aims at knowledge to actually create them. Research in the engineering sciences thus results in scientific models of the imagined phenomena. These models must be such that they make it possible to actually create the imagined phenomena with technological means (Boon 2017).
} 
issues (a-f) that an epistemology of models needs to address, and suggests that dealing with these issues requires taking into account the construction of models instead of starting from ready-made models. A recently published method for (re)constructing scientific models (Boon 2020) is taken as an example for this purpose (see note 19). According to this method, models initially form hubs in which heterogeneous aspects are brought together and integrated into a coherent whole. This account of how scientific models are constructed agrees with various of the insights developed in the present article. In particular, the method assumes that the construction of a scientific model involves the identification and conceptual articulation of the (imagined) targetphenomenon. Furthermore, the method requires specifying the physical or technological conditions that are considered relevant to the target-phenomenon, together with the measurable variables that characterize it, which complies with the proposed explanation of how the imagined phenomenon is connected with the real-world phenomenon.

It is explored how the construction of models according to this method of construction sheds light on the philosophical issues (a-f) relevant for an epistemology of scientific models, based on which several conclusions can be drawn: (1) The method of construction shows that scientific models are not somehow read from reality (like a photograph or drawing). (2) The insight that constructing a model involves combining heterogeneous aspects makes it clear that both non-linguistic and conceptually meaningful linguistic content is selected and built into the model. This implies that the scientific model is not merely a (non-linguistic) picture or graph or set of mathematical equations that somehow literally represents the (objective) structure of a real-world target-phenomenon. Instead, the model is more like a coherent story that contains linguistic and non-linguistic content presented in, for example, a scientific article. Moreover, the way a scientific model is constructed depends on contextual information, which is related to the specificities of the discipline and to the epistemic purpose of the research project. Therefore, it makes sense to assume that a scientific article in its entirety presents the scientific model. (3) The method of construction explains how it is possible to connect between the model and the purported non-observable real-world phenomenon. This accords with my take on the semantic view in which the role of measurable variables is critical. However, the conceptual articulation of the phenomenon is also crucial, in particular to enable different types of inferential reasoning by means of the model. This requires to also explain how conceptually meaningful content enters the model. (4) In Section 4 it is argued that the target-phenomenon is not 'readymade,' but requires conceptual articulation. In line with this insight, Section 5 explains that, according to the method of construction, the identification of the phenomenon is an inherent part of the construction process. The conception of the target-phenomenon (i.e., the imagined phenomenon) and the model are therefore co-constructed. For example, the construction of a model starts with observations, experiences, or data that a researcher wants to deal with, and is often also related to a broader (practical or theoretical) problem. In conceptual articulation, these observations, experiences, or data are brought under (scientific) concepts (i.e., 'subsumption under concepts'). This is one way how conceptual content enters the model. (5) The method of construction explains how a model is justified. I distinguish three ways that complement each other. First, there is the comparison between model outcomes and experimental data (as in the semantic view). But it must also be assessed whether the model meets relevant epistemic and pragmatic criteria. 'Internal coherence,' for example, warrants that the 
model enables inferential reasoning. In addition, the construction of a model requires that the choices and decisions that are made with regard to the various elements built into the model be justified. (6) Lastly, therefore, it is argued that an epistemology of scientific models should also explain and justify the choices and decisions from researchers on aspects that must be built into the model. Moreover, researchers need to somehow interpret empirical and experimental findings (e.g., subsumption under concepts to imagine the target-phenomenon), for which intellectual capabilities of researchers are crucial, such as imagination. ${ }^{43}$ Therefore, in accordance with the transcendental and pragmatic approach adopted here, an epistemology of models requires further clarification as to how the choices, decisions and conceptualizations by researchers are made possible. To explain this in more depth, I claim that the construction of scientific models is enabled and guided, but also restricted, by the disciplinary perspective within which researchers work. Section 5, "Taking Stock" summarizes the aspects of an epistemology of scientific models in a number of statements.

The second part of this article concerns the role of (disciplinary) perspectives in developing an epistemology of models. The approach is again transcendental and pragmatic. I side with Massimi and McCoy (eds. 2020, 4) who state that: "[U]ltimately it does not matter how one defines the notion of "scientific perspective" (e.g., à la Giere, or à la van Fraassen, among others; with reference to scientific models, Kuhnian paradigms, or concepts and conceptual schemes). What matters most is what perspectivism can achieve, how it enters the practice of science, the challenges it poses, and the solutions it offers." Nonetheless, I take it that it needs to be explained why we need disciplinary perspectives in an epistemology of scientific models. According to Massimi and McCoy, the practice orientation stresses the human point of view and therefore the role of perspectives. Similarly, my philosophical argument for defending the indispensable role of perspectives is based on the conclusion that the philosophically problematic representational relationship between model and target, and issues that arise from giving up the picture-metaphor of representation, must be clarified by explaining how it is possible that researchers construct scientific models, and how it is possible that these models can be used for inferential reasoning in performing epistemic tasks.

Section 6, therefore, explains the epistemological and pragmatic roles of (disciplinary) perspectives in the construction of scientific models. It starts from asking: What are perspectives, and why do we need them? First, I argue that this is because perspectives in the broad sense enable and constrain the epistemic activities of researchers when constructing and using scientific models. Building on Giere's (2006) work on perspectives in science, I distinguish five types of perspectives and explain their roles with regard to the issues addressed in the first part of this article.

\footnotetext{
${ }^{43}$ The role of imagination introduced here goes beyond its role in the idea of models as fiction, as in Barberousse and Ludwig (2008), who investigate what it means to say that 'models are fictions,' and claim that the role of models in scientific practice lies in the activity of imagining. According to them, models are artifacts that enable researchers to play and experiment with ideas. In part, I agree with their idea in the sense that their notion of imagination is more or less synonymous with the notion of inferential reasoning that I use. But, by interpreting models as fictions, they avoid the philosophical premise that models must be understood primarily in terms of a putative referential relationship between the model and the purported 'real-world' phenomenon. In contrast, I aim at an epistemology of models that also explains how models make inferential reasoning about the real-world phenomena possible. In addition, I stress that imagination plays a role in the construction of models and the conception of phenomena.
} 
Then, I suggest that these different types of perspectives are part of the disciplinary perspective of a discipline. Next, I propose a Kuhnian framework for characterizing disciplinary perspectives in the form of a preliminary scheme that lists elements typically included in disciplinary perspectives. In short, the Kuhnian framework consists of a coherent set of heterogeneous elements, which includes the types of problems, phenomena, fundamental and ontological beliefs, measurement techniques, experimental procedures, mathematical, theoretical and conceptual frameworks, investigative strategies, and representational means that are typical of the discipline. The elements in the Kuhnian framework partly reflect those in the method for constructing models. This coherence explains why disciplinary perspectives understood in this way make the (discipline-specific) construction of scientific models possible and also limit it.

Finally, it needs to be explained how to deal with cherished values such as objectivity, which in more traditional views of science requires "a view from nowhere" (Massimi and McCoy eds., 2020, 2). Objectivity seems to require that knowledge is not constructed through perspectives. I defend that disciplinary perspectives are indispensable for the construction of models These contributions from researchers are indelibly built into the resulting scientific model and makes it 'discipline-specific,' rather than objective. To deal with this challenging issue and prevent harmful subjectivism, my suggestion is that in scientific research, disciplinary perspectives can and should be made explicit and critically evaluated, for which the Kuhnian framework proposed here may prove helpful.

Acknowledgements An earlier version of this paper has been presented at the Models and Simulation conference (MS8, 2018, University of South Carolina, Columbia, South Carolina). This work is financed by an Aspasia grant (409.40216) of the Dutch National Science Foundation (NWO) for the project Philosophy of Science for the Engineering Sciences. I wish to thank Michaela Massimi, Henk Procee and two anonymous reviewers for constructive suggestions.

Open Access This article is licensed under a Creative Commons Attribution 4.0 International License, which permits use, sharing, adaptation, distribution and reproduction in any medium or format, as long as you give appropriate credit to the original author(s) and the source, provide a link to the Creative Commons licence, and indicate if changes were made. The images or other third party material in this article are included in the article's Creative Commons licence, unless indicated otherwise in a credit line to the material. If material is not included in the article's Creative Commons licence and your intended use is not permitted by statutory regulation or exceeds the permitted use, you will need to obtain permission directly from the copyright holder. To view a copy of this licence, visit http://creativecommons.org/licenses/by/4.0/.

\section{References}

Bailer-Jones, D. M. (2009). Scientific models in philosophy of science. Pittsburgh: University of Pittsburgh Press.

Barberousse, A., \& Ludwig, P. (2008). Models as fictions. Chapter 4. In M. Suárez (Ed.), Fictions in science: Philosophical essays on modeling and idealization (pp. 64-82). New York: Routledge.

Bogen, J. (2011). 'Saving the phenomena' and saving the phenomena. Synthese, 182(1), 7-22. https://doi. org/10.1007/s11229-009-9619-4.

Bogen, J., \& Woodward, J. (1988). Saving the phenomena. The Philosophical Review, 97(3), 303-352. https://doi.org/10.2307/2185445. 
Bokulich, A. (2009). Explanatory fictions. In M. Suárez (Ed.), Fictions in science: Philosophical essays on modeling and idealization (pp. 91-109). New York: Routledge.

Boon, M. (2012). Scientific concepts in the engineering sciences: Epistemic tools for creating and intervening with phenomena. In U. Feest \& F. Steinle (Eds.), Scientific concepts and investigative practice (pp. 219243). Berlin: De Gruyter.

Boon, M. (2017). Measurements in the engineering sciences: An epistemology of producing knowledge of physical phenomena. In N. Mößner \& A. Nordmann (Eds.), Reasoning in measurement (pp. 203-219). London and New York: Routledge.

Boon, M. (2020). Scientific methodology in the engineering sciences. Chapter 4. In D. Michelfelder \& N. Doorn (Eds.), Routledge handbook of philosophy of engineering. Routledge. In press.

Boon, M., \& Knuuttila, T. (2009). Models as epistemic tools in engineering sciences: A pragmatic approach. In A. Meijers (Ed.), Philosophy of technology and engineering sciences. Handbook of the philosophy of science (Vol. 9). Elsevier/North-Holland: 687-720.

Boon, M., \& Van Baalen, S. (2019). Epistemology for interdisciplinary research-shifting philosophical paradigms of science. European Journal for Philosophy of Science, 9(1), 16. https://doi.org/10.1007 /s13194-018-0242-4.

Chang, H. (2014). Epistemic activities and Systems of Practice: Units of analysis in philosophy of science after the practice turn. In L. Soler, S. Zwart, M. Lynch, \& V. Israel-Jost (Eds.), Science after the practice turn in the philosophy, history and social studies of science (pp. 67-79). London and Abingdon: Routledge.

Chang, H. (2020). Pragmatism, Perspectivism, and the historicity of science. Chapter 1. In M. Massimi \& C. D. McCoy (Eds.), Understanding Perspectivism: Scientific challenges and methodological prospects (pp. 10-28). Routledge: Routledge Studies in the Philosophy of Science.

Contessa, G. (2010). Scientific models and fictional objects. Synthese, 172(2), 215-229.

Fagan, M.B. (2020). Explanation, Interdisciplinarity, and perspective. Chapter 3. In M. Massimi \& C. D. McCoy (Eds.), Understanding Perspectivism: Scientific challenges and methodological prospects (pp. 28-48). Routledge: Routledge Studies in the Philosophy of Science.

Feest, U. (2010). Concepts as tools in the experimental generation of knowledge in cognitive neuropsychology. Spontaneous Generations, 4(1), 173-190.

Fine, A. (1993). Fictionalism. Midwest Studies in Philosophy, 1993(18), 1-18 Reprinted in: M. Suárez (ed., 2009): 19-36.

Frigg, R. \& Hartmann, S. (2018). Models in science, The Stanford Encyclopedia of Philosophy Spring 2020 Edition. E.N. Zalta (ed.), URL =https:/plato.stanford.edu/archives/sum2018/entries/models-science/. Accessed 5 Aug 2015

Frigg, R., \& Nguyen, J. (2016a). The fiction view of models reloaded. The Monist, 99(3), 225-242.

Frigg, R. \& Nguyen, J. (2016b). Scientific representation. The Stanford Encyclopaedia of Philosophy Winter 2016 Edition. E.N. Zalta (ed.), URL = <https://plato.stanford.edu/archives/win2016/entries/scientificrepresentation/>. Accessed 5 Aug 2015

Giere, R. N. (1999). Science without Laws. Chicago: University of Chicago Press.

Giere, R. N. (2002). How models are used to represent reality. http://philsci-archive.pitt.edu/archive/00000838/.

Giere, R. N. (2006). Scientific perspectivism. Chicago: The University of Chicago Press.

Giere, R. N. (2010). An agent-based conception of models and scientific representation. Synthese, 172(2), 269-281. https://doi.org/10.1007/s11229-009-9506-z.

Glymour, B. (2002). Data and phenomena: A distinctions reconsidered. Erkenntnis, 52, 29-37.

Hacking, I. (1983). Representing and intervening: Introductory topics in the philosophy of natural science. Cambridge: Cambridge University Press.

Hacking, I. (1992). The self-vindication of the laboratory sciences. In Science as Practice and Culture. A. Pickering (pp. 29-64). Chicago: University of Chicago Press.

Knuuttila, T., \& Boon, M. (2011). How do models give us knowledge? The case of Carnot's ideal heat engine. European Journal for Philosophy of Science, 1(3), 309-334. https://doi.org/10.1007/s13194-011-0029-3.

Massimi, M. (2007). Saving unobservable phenomena. Britisch Journal Philosophy of Science., 58, $235-262$.

Massimi, M. (2008). Why there are no ready-made phenomena: What philosophers of science should learn from Kant. Royal Institute of Philosophy Supplement., 63, 1-35. https://doi.org/10.1017 /S1358246108000027.

Massimi, M. (2011). From data to phenomena: A Kantian stance. Synthese., 182, 101-116. https://doi. org/10.1007/s11229-009-9611-z. 
Massimi, M. (forthcoming). Two kinds of exploratory models. Philosophy of Science. Proceedings of the PSA 2018, Seattle.

Massimi, M., \& McCoy, C. D. (Eds.). (2020). Understanding Perspectivism: Scientific challenges and methodological prospects. Routledge: Routledge Studies in the Philosophy of Science.

Mitchell, S.D. (2020). Perspectives, representation, and integration. Chapter 10. In M. Massimi \& C. D. McCoy (Eds.), Understanding Perspectivism: Scientific challenges and methodological prospects (pp. 178-193). Routledge: Routledge Studies in the Philosophy of Science.

Morgan, M. S., \& Morrison, M. (Eds.). (1999). Models as mediators - perspectives on natural and social science. Cambridge: Cambridge University Press.

Morrison, M. (1999). Models as autonomous agents. Chapter 3. In M. S. Morgan \& M. Morrison (Eds.), Models as Mediators - Perspectives on Natural and Social Science (pp. 38-65). Cambridge: Cambridge University Press.

Morrison, M., \& Morgan, M. S. (1999). Models as mediating instruments. Chapter 2. In M. S. Morgan \& M. Morrison (Eds.), Models as mediators - Perspectives on natural and social science (pp. 10-37). Cambridge: Cambridge University Press.

Nersessian, N. (2009a). Model-based reasoning in interdisciplinary engineering. The handbook of the philosophy of technology \& engineering sciences, 687-718. https://doi.org/10.1016/B978-0-444-516671.50031-8.

Nersessian, N. J. (2009b). How do engineering scientists think? Model-based simulation in biomedical engineering research laboratories. Topics in Cognitive Science, 1(4), 730-757. https://doi.org/10.1111 j.1756-8765.2009.01032.x.

Nersessian, N. J. (2009c). Creating scientific concepts. Cambridge: MIT Press.

Nersessian, N. J., \& Patton, C. (2009). Model-based reasoning in interdisciplinary engineering. In A. Meijers (Ed.), Handbook of the philosophy of technology and engineering sciences (pp. 687-718). Amsterdam: Elsevier.

Rice, C. (2020). Universality and the problem of inconsistent models. Chapter 5. In M. Massimi \& C. D. McCoy (Eds.), Understanding Perspectivism: Scientific challenges and methodological prospects (pp. 85-108). Routledge: Routledge Studies in the Philosophy of Science.

Rouse J. (2009). Laboratory fictions. Chapter 3 in: Suárez, M. (ed. 2009): 45-63.

Rouse, J. (2011). Articulating the world: Experimental systems and conceptual understanding. International Studies in the Philosophy of Science, 25(3), 243-254. https://doi.org/10.1080/02698595.2011.605246.

Rueger, A. (2005). Perspectival models and theory unification. The British Journal for the Philosophy of Science, 56(3), 579-594. https://doi.org/10.1093/bjps/axi128.

Suárez, M. (2003). Scientific representation: Against similarity and isomorphism. International Studies in the Philosophy of Science., 17, 225-244.

Suárez, M. (2004). An inferential conception of scientific representation. Philosophy of Science, 71, 767-779.

Suárez, M. (Ed.) (2009a). Fictions in science: Philosophical essays on modeling and idealization. New York: Routledge.

Suárez, M. (2009b). Fictions in scientific practice. In Suárez (Ed.), Fictions in science: Philosophical essays on modeling and idealization (pp. 3-15). New York, Routledge.

Suárez, M. (2012). The ample modelling mind. Studies in History and Philosophy of Science, 43, $213-217$.

Suppe, F. (1989). The semantic conception of theories and scientific realism. Urbana and Chicago: University of Illinois Press.

Teller (2020). What is perspectivism, and does it count as realism? Chapter 3. In M. Massimi \& C. D. McCoy (Eds.), Understanding Perspectivism: Scientific challenges and methodological prospects (pp. 49-65). Routledge: Routledge Studies in the Philosophy of Science.

Toon, A. (2012). Similarity and scientific representation. International Studies in the Philosophy of Science, 26(3), 241-257. https://doi.org/10.1080/02698595.2012.731730.

Vaihinger, H. (1911). The philosophy of 'as if'. German original. English translation: London: Kegan Paul 1924.

Van Fraassen, B. C. (1980). The scientific image. Oxford: Clarendon Press.

Van Fraassen, B. C. (2008). Scientific representation. Oxford: Oxford University Press.

Wolff, J. E. (2020). Representationalism in measurement theory. Structuralism or perspectivalism? Chapter 6. In M. Massimi, \& C. D. McCoy (Eds.), Understanding Perspectivism: Scientific challenges and methodological prospects (pp. 109-126). Routledge: Routledge Studies in the Philosophy of Science. 
Woodward, J. F. (2011). Data and phenomena: A restatement and defense. Synthese, 182(1), 165-179. https://doi.org/10.1007/s11229-009-9618-5.

Publisher's note Springer Nature remains neutral with regard to jurisdictional claims in published maps and institutional affiliations.

\section{Affiliations}

\section{Mieke Boon ${ }^{1}$}

1 Department of Philosophy, University of Twente, PO Box 217, 7500 AE Enschede, The Netherlands 